\title{
One-Ampere Conductor Method for Tubular Linear Induction Motor for Size Reduction of Primary Iron Core
}

\author{
Byeong-Hwa Lee ${ }^{1}$, Kyu-Seob Kim ${ }^{1}$, Soon-O Kwon ${ }^{1}$, Tao Sun ${ }^{1}$, Jung-Pyo Hong ${ }^{1}$, , and Jung-Ho Lee ${ }^{2}$ \\ ${ }^{1}$ Department of Automotive Engineering, Hanyang University, Seoul 133-791, Korea \\ ${ }^{2}$ Department of Electrical Engineering, Hanbat National University, Daejeon 305-719, Korea
}

(Received 14 October 2010, Received in final form 26 February 2011, Accepted 2 March 2011)

\begin{abstract}
This paper presents size reduction of primary iron core for tubular linear induction motor by improved winding configuration. Using one-ampere conductor method, magnetic field analysis of tubular linear induction motor for size reduction is conducted. Size reduction and improvement of air gap flux distribution is achieved by improved winding configuration, and analysis results are verified by finite element analysis (FEA) and experiments.
\end{abstract}

Keywords : analytical method, finite element analysis, magnetic flux density, one-ampere conductor method, tubular linear induction motor

\section{Introduction}

Rotary type induction motors are generally used in industrial machines but applying rotary type motor to linear motion system is limited. Additional mechanical loss is generated by mechanical friction such as gear, ball screw and belt using rotary motor in linear moving systems, then complicated circuit composition problem occurs and efficiency in economical point of view is not reasonable. Various designs of linear motors are studied to overcome such limitations of rotary machines. One of them is the short primary member tubular linear induction motor (TLIM) [1, 2]. Fig. 1 shows the structure of TLIM. The primary core in TLIM is made of several disc or transversal lamination of ferromagnetic material. The main advantages of the TLIM are that it is rugged and easy to build. Because of TLIM is used at a very low speed, the dynamic end effects are negligible and the static end effect can be minimized by good design [3, 4].

The fundamental principle of thrust force generation in linear magnetic motor is generally identical to common rotary type motor, however the length of primary iron core is limited. Therefore distribution of ideal winding is not possible, even with ideal distributed winding, the flux distribution in the air gap placed in the ends of primary

*Corresponding author: Tel: +82-2-2220-4466

Fax: +82-2-2220-4465, e-mail: hongjp@hanyang.ac.kr iron core is lower than magnetic flux density in the air gap placed of the primary core center [5].

An improved winding configuration is introduced, which improve magnetic flux density distribution in the primary core ends and leads to the size reduction. By shifting single layer of conductors, double layer of conductors are constructed and total length of primary core is reduced with improved magnetic flux density distribution.

For the magnetic field calculation, one-ampere conductor method is introduced and verified by finite element method using axi-symmetry analysis and experiments.

\section{Analysis Theory}

2.1. Field calculation by one ampere conductor Magnetic flux density distribution in the air gap is the
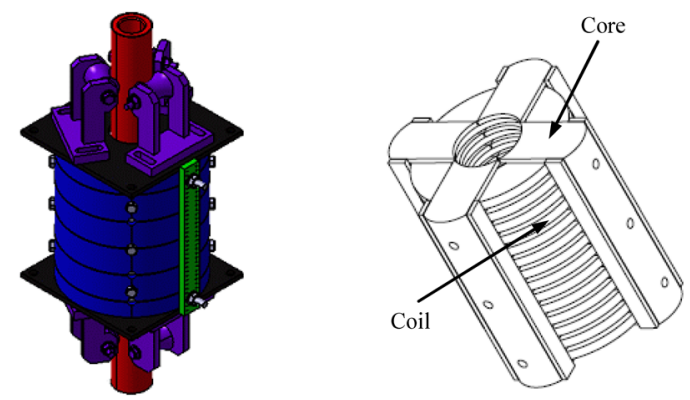

Fig. 1. Structure of TLIM. 


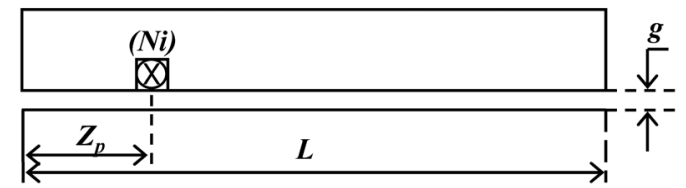

(a) Current conductor distribution

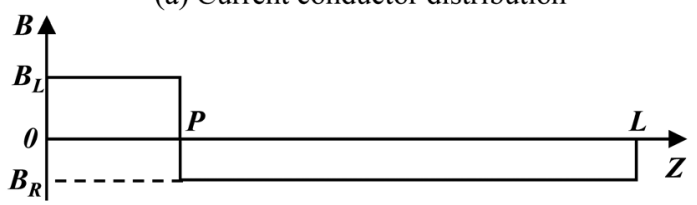

(b) Air-gap current density

Fig. 2. Air-gap flux density distribution by one ampere conductor element.

most important motor parameter. In order to calculated air gap magnetic flux density distribution, flux distribution by one conductor element corresponding to unit excitation of stator magneto motive force is calculated then total magnetic flux density distribution is calculated considering space and time distribution of total conductor. Assumptions for analysis are;

1) Relative permeability of primary and secondary core are infinite, also there are no saturation in the cores.

2) Fringing effect of magnetic flux is not considered.

3) Conductivity of secondary reaction plate and secondary conductor are zero

4) Primary core except for place that conductor is located is smooth core which is corrected by carter coefficient.

Fig. 2 presents the concept of one-ampere conductor method and flux distribution. Magnetic flux density distribution can be calculated by equation (4) with given geometry in Fig. 2. In Fig. 2(a) total reluctance is shown following equation (1) when reluctances of left and right side of $Z_{P}$ are relatively defined as $R_{L}$ and $R_{R}$.

$$
\begin{aligned}
R & =R_{L}+R_{R} \\
& =\frac{g}{\mu_{o} Z_{p}}+\frac{g}{\mu_{o}\left(L-Z_{p}\right)}
\end{aligned}
$$

where $g$ is mechanical clearance.

On the other hand, if cross-section area of flux path is $S$, corresponding path is expressed by equation (2). Therefore, magnetic flux density of left and right side by the standards of $Z_{P}, B_{L}$ and $B_{R}$, is expressed by equation (3) and (4) [6].

$$
\begin{aligned}
F & =N I=R \Phi \\
& =\mathrm{R}(\vec{B} \cdot \vec{S}) \\
B_{L} & =\mu_{o} \frac{N i}{g} \times \frac{L-Z_{p}}{L}
\end{aligned}
$$

$$
B_{R}=-\mu_{o} \frac{N i}{g} \times \frac{Z_{p}}{L}
$$

\subsection{Field calculation by multiple conductors}

In order to consider slot effect, Carter coefficient is used.

$$
\begin{aligned}
& g^{\prime}=g_{o} K_{c}, g_{o}=K_{c}(g+d), \\
& K_{c}=\frac{t_{s}\left(5 g_{o}+w_{s}\right)}{t_{s}\left(5 g_{o}+w_{s}\right)-w_{s}^{2}}
\end{aligned}
$$

where, $d$ is thickness of reaction plate, $t_{s}$ is slot pitch, $w_{s}$ is tooth width.

Air gap magnetic flux density by two current carrying conductors is calculated by superposition of air gap magnetic flux density due to each current carrying conductor with equation (3) and (4).

When $n$ - conductors are distributed in the slots along $L$, magnetic flux density in the air gap can be expressed as equation (5).

$$
\begin{aligned}
B_{p}(z) & =\sum_{k=1}^{m} B_{L K}+\sum_{k=m+1}^{s n} B_{R K} \\
& =-\frac{\mu_{0}}{g^{\prime} L} \sum_{k=1}^{m}(N i)_{k} z_{k}+\frac{\mu_{0}}{g^{\prime} L} \sum_{k=m+1}^{s n}(N i)_{k}\left(L-z_{k}\right)
\end{aligned}
$$

where, $m$ is phase number, $s n$ is slot number respectively. Applying for Fourier series expansion to magnetic flux density by single conductor,

$$
B_{p}(z, t)=\sum_{k=1}^{\infty}\left(\frac{2 \mu_{0} N i(t)}{h \pi g^{\prime}} \sin \frac{h \pi}{L} z_{p}\right) \cos \frac{h \pi}{L} z
$$

where, $h$ is harmonic order.

Then magnetic flux density distribution due to total conductors is

$$
B_{p}(z, t)=\sum_{k=1}^{\infty}\left(\sum_{p=1}^{s n} \sum_{v=1}^{m}\left(\frac{2 \mu_{0} N i_{v}(t)}{h \pi g^{\prime}} \sin \frac{h \pi}{L} z_{p}\right)\right) \cos \frac{h \pi}{L} z
$$

\subsection{Force calculation}

Analytical solutions for force calculations are well established in previous researches equation $(9)[1,2]$.

$$
F_{z}=-a_{e} \mu_{0} d \operatorname{Re}\left[\int_{0}^{\infty} \bar{J}_{2}(z) \cdot \bar{H}(z)^{*} d z\right]
$$

where, $a_{e}$ is effective stack length of primary part, $\bar{J}_{2}(z)$ is eddy current density in reaction, $\bar{H}(z)^{*}$ is complex conjugate of resultant magnetic flux density.

Normal force consists of attraction and repulsion force. Attraction and repulsion force can be calculated by equation (10) and (11). Resultant normal force is equation (12). 


$$
\begin{aligned}
F_{a n}= & -a_{e} \mu_{0}\left[\int_{0}^{L}\left|\bar{H}(z)^{2}\right| d z\right] \\
F_{n r}= & -a_{e} \mu_{0} d \operatorname{Re}\left[\int_{0}^{L} J_{n f} \exp \left(-j \beta_{n} z\right)\right. \\
& \left.+J_{n b} \exp \left(j \beta_{n} z\right)^{*} d z\right] \\
F_{n}= & F_{a n}+F_{n r}
\end{aligned}
$$

\section{Improvement of Winding Configuration}

\subsection{Analysis model}

General winding method of TLIM is single layer of conductors at the end region and double layer at the other regions. In addition, magnetic flux density at the each side of end region decreases.

Fig. 3 shows the concept of size reduction with stator winding variation. Fig. 3(a) shows the general 15 slot TLIM, and Fig. 3(b) shows the method. Winding distribution in both stator ends, c-phase winding placed can be replaced by the sum of A-phase and B-phase conductor. Therefore, two slots in the stator end region become empty. Then, single layer of both stator ends filled with A-phase and B phase. Finally, empty slots are removed and stator length is reduced. In addition, flux densities in the stator end regions are improved.

One-ampere conductor method is used for the calcula-

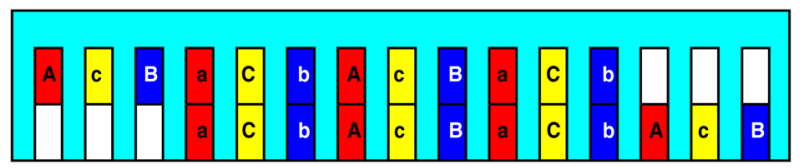

(a) Winding configuration of 15 slot model

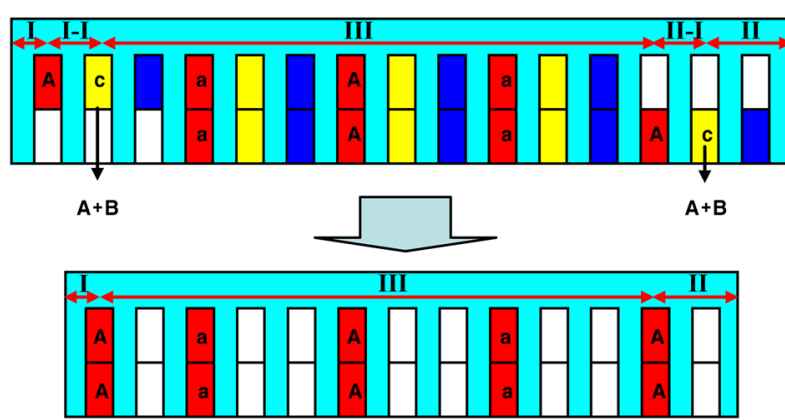

(b) Reduction of stator slots number from 15 to 13

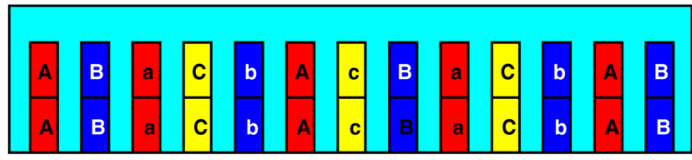

(c) 13 slot model

Fig. 3. Concept of stator size reduction and winding distribution.

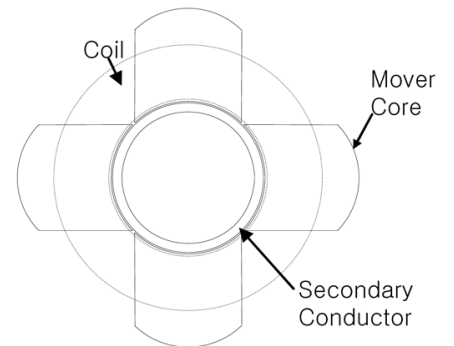

(a) Top view of 13 slot and 15 slot

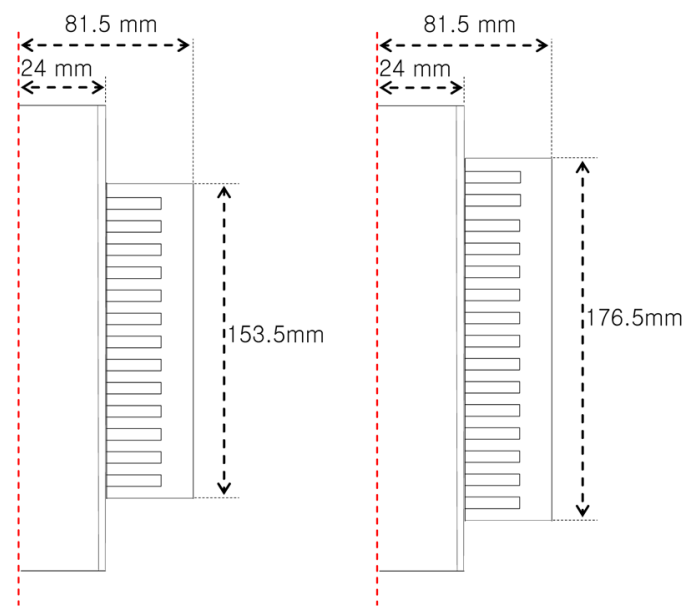

(b) Side view of 13 slot and 15 slot

Fig. 4. Dimension of 13 slot and 15 slot model.

Table 1. shows the specifications of analysis model.

\begin{tabular}{cc}
\hline Items & Values \\
\hline Pole number / Slot number & $4 / 15$ \\
Phase number & 3 \\
Input frequency $(\mathrm{Hz})$ & 7 \\
External diameter / Internal diameter $(\mathrm{mm})$ & $163.0 / 53$ \\
Stack length $(\mathrm{mm})$ & 176.5 \\
Air-gap length $(\mathrm{mm})$ & 2.5 \\
Turn number per a phase & 76 \\
Thickness of secondary conductor $(\mathrm{mm})$ & 2.0 \\
\hline
\end{tabular}

tion. Fig. 4(a) and Fig. 4(b) show top view and the dimension of prototype model and improved model.

Table 1 shows the specifications of analysis model.

\section{Verification}

\subsection{Verification by FEA}

Fig. 5 shows the flux distribution of 13 slot model from FEA. Fig. 6 and Fig. 7 show the comparison of air gap magnetic flux density from FEA and one-ampere conductor method (ACM) of 13 slot and 15 slot model. In the analysis, balanced 3-phase is assumed and corresponding phase current is applied. Up to $10^{\text {th }}$ harmonic is considered for magnetic flux density calculation in the ACM at 0 


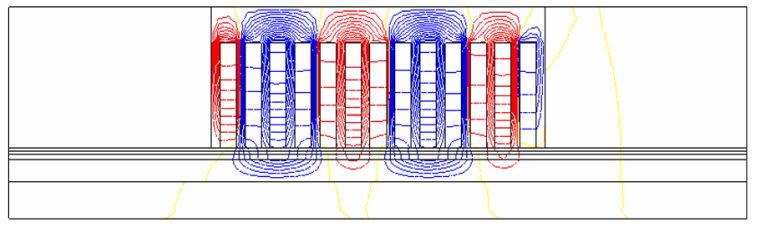

Fig. 5. Flux distribution of 13 slot model.

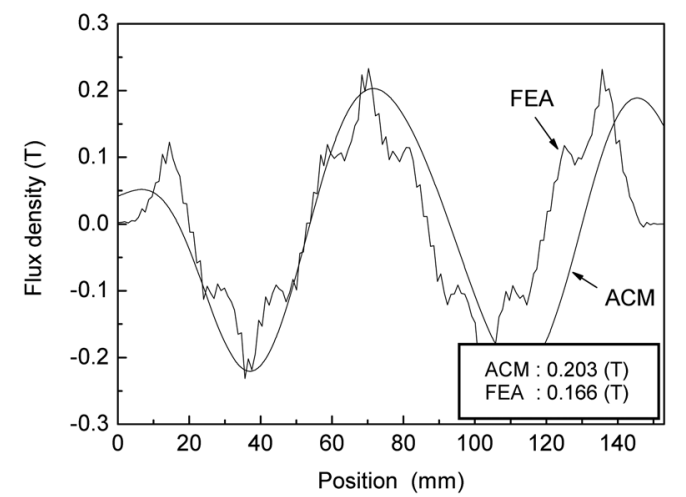

(a) Air gap magnetic flux density of 13 slot model at $w t=0^{\circ}$

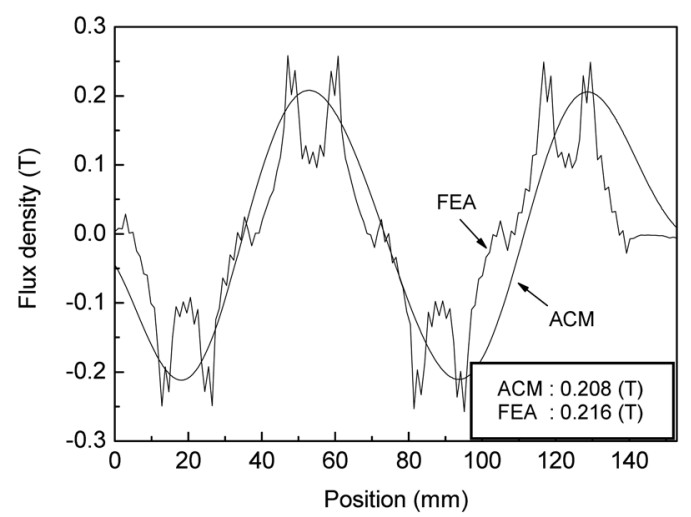

(b) Air gap magnetic flux density of 13 slot model at $\mathrm{wt}=90^{\circ}$

Fig. 6. Air gap magnetic flux density of 13 slot model.

slip according to phase. From equation (8), air gap magnetic flux density wave form is dependent to harmonic number, as harmonic order increased, magnetic flux density waveform reaches to rectangular form. Therefore, appropriate number of harmonic component should be chosen. In the analysis results, up to $10^{\text {th }}$ harmonics are considered. Considering simplicity, presented analysis method is very effective.

\subsection{Verification by measurements}

According to improved winding method, turn number per a phase and interval of coil are different. Thus, voltage source analysis is necessary due to unbalance of impedance of phase compared with general induction motor. However, in case of analysis model, motor is operated at low speed, therefore, unbalance of phase current is reduced.

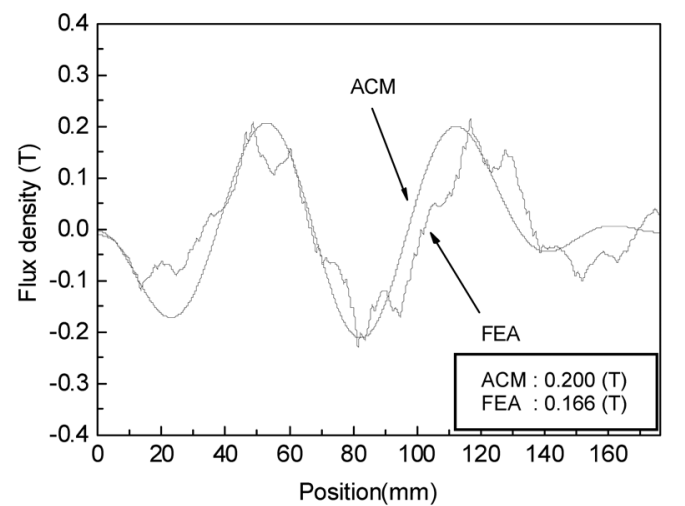

(a) Air gap magnetic flux density of 15 slot model at wt $=0^{\circ}$

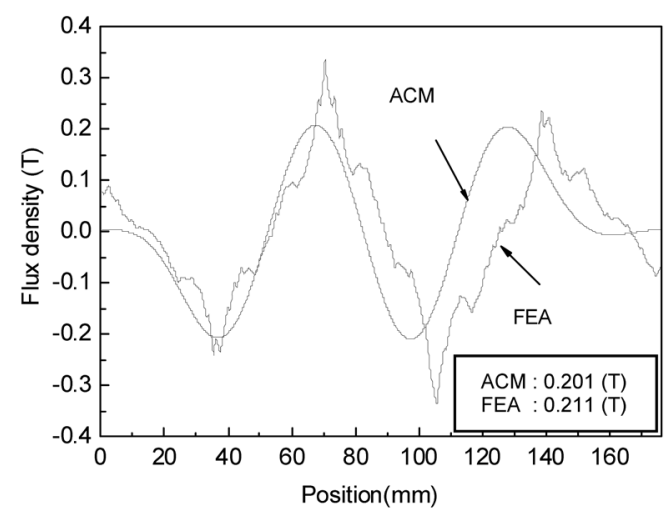

(b) Air gap magnetic flux density of 15 slot model at $w t=90^{\circ}$

Fig. 7. Air gap magnetic flux density of 15 slot model.

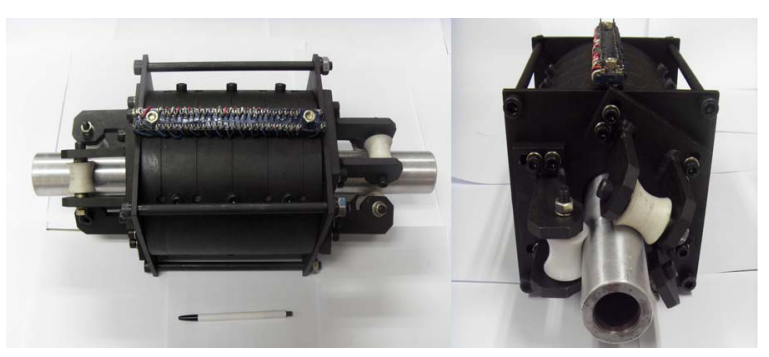

Fig. 8. Fabricated TLIM.

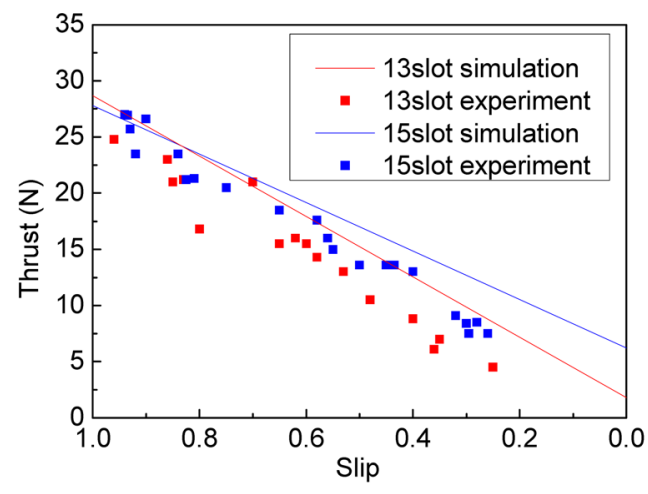

Fig. 9. Comparison of thrust force according to slip. 
Therefore, analysis is conducted with current source analysis.

Fig. 8 shows fabricated 13 slot model motor. In case of 13slot model, improvement of magnetic flux density is found in the core ends, while little difference is found in core center.

Fig. 9 shows the thrust force comparison of 13 and 15 slot models. Measurements are conducted with dc power supply calculated values follows the experiments well. In the high slip region, improvements are found in 13 slot model, however, in the low slip region, 15 slot model has better performance. Considering further performance improvement from optimal design, it is expected that 13 slot model can provide better performance than 15 slot models with reduced stator size.

\section{Conclusion}

In this paper, simple calculation method for magnetic flux density distribution; one-ampere conductor method is introduced and winding configuration for size reduction is presented. With one-ampere conductor method, magnetic flux density distribution of tubular linear induction motor is effectively estimated. Suggested improved winding configuration enables to reduce stator size from conventional windings. Comparative analysis on resultant air gap magnetic flux density distribution was carried out. Analysis results are verified by FEA with axi-symmetric formulation and experiments.

\section{References}

[1] I. Boldea and S. A. Nasar, Linear Motion Electromagnetic System, Wiley, New York (1976).

[2] B. T. Ooi and D. C. White, IEEE Trans. Power Apparatus Syst. 89, 638 (1970).

[3] S.-M. Jang, J.-Y. Choi, H.-W. Cho, and S.-H. Lee, KIEE International Transaction on Electrical Machinery and Energy Conversion Systems 5B, 154 (2005).

[4] D. Deas, P. Kuo-Peng, N. Sadowski, A. M. Oliveira, J. L. Roel, and J. P. A. Bastos, IEEE Trans. Magn. 38, 1165 (2002).

[5] R. Haghmaram and A. Shoulaie, "Study of Traveling Wave Tubular Linear Induction Motors", International Conference on Power System Technology (2004).

[6] W. J. Jeon, M. Tanabiki, T. Onuki, and J. Y. Yoo, "RotaryLinear Induction Motor Composed of Four Primaries with Independently Energized Ring-Windings", IEEE Industry Application Society Annual Meeting (1997). 